\title{
Trapped Bose gases with large positive scattering length
}

\author{
M. Thøgersen, D.V. Fedorov, And A.S. Jensen \\ IFA, Aarhus University, 8000 Aarhus C, Denmark \\ PACS \\ $-03.75 . \mathrm{Hh}, 03.75 . \mathrm{Nt}, 21.45 .+\mathrm{v}$
}

\begin{abstract}
We calculate the energy and the condensate fraction of a system of trapped bosons interacting via a short-range two-body potential with positive scattering length. The potential is attractive and has a two-body bound state. When the scattering length is small compared to the trap length the system is model independent: all potential models - attractive, repulsive and zero-range - provide similar results. When the scattering length is large the attractive model differs qualitatively from the repulsive and zero-range models. In this regime the system with attractive potential becomes independent of the scattering length, with both the energy and the condensate fraction converging towards finite constants.
\end{abstract}

Introduction. - The density of trapped cold gases is generally low under typical experimental conditions [1], such that the parameter $n b^{3}$ is small, where $n$ is the particle density and $b$ is the range of the inter-particle potential. In other words the typical distance between particles is much larger than the range of the potential, and the typical relative momentum between particles is much smaller ' than the inverse range of the potential.

In this regime the system of particles exhibits universality (also called model independence or shape independence) [2]. The system is not sensitive to the details of the potential and the properties of the system are essentially determined by only few low-energy parameters of the potential. Very different interaction models then provide quantitatively similar results as soon as the low-energy parameters are the same.

In a two-body system the universality is manifested in the well known effective range expansion, where the lowenergy $s$-wave phase shift $\delta$ is determined by only two parameters, the scattering length $a$ and the effective range $r_{e}$

$$
k \cot \delta=-\frac{1}{a}+\frac{1}{2} r_{e} k^{2}+O\left(r_{e}^{3} k^{4}\right)
$$

where $k$ is the relative momentum between particles. The effective range $r_{e}$ is typically of the order of the range of the potential while the scattering length can vary greatly.

In three-body systems the universality manifests itself in the Thomas [3] and Efimov [4] effects, the Phillips line [5], and other low-energy phenomena [6], also in two dimensions [7].

Cold gases also exhibit universality: in the dilute limit their properties, in particular the energy per particle, are independent on the shape of the inter-particle potential and are determined by the scattering length alone. This universality is customarily employed by using the zerorange (pseudo-)potential for theoretical descriptions of the Bose-Einstein condensates. The zero-range potential has only one parameter, the scattering length. Combined with the Hartree-Fock product wave-function the zero-range potential model is known as the Gross-Pitaevskii equation $[8]$.

The scattering length in a trapped atomic gas can be tuned to an essentially arbitrary value using the technique of Feshbach resonances [1]. This gives a possibility to investigate trapped systems with very large scattering lengths. In this case the first term in the effective range expansion eq. (1) vanishes and the system becomes sensitive to the effective range of the potential.

The limits of the zero-range model have been tested by numerical calculations with finite-range potentials. In particular, for the large positive scattering length repulsive potentials have been employed within Monte-Carlo methods [9-11]. These investigations showed that as the scattering length is increased the energy of the Bose gas with a repulsive potential exceeds the zero-range predictions and the condensate fraction becomes considerably depleted.

However, repulsive potentials have a problem when modelling an increasingly large positive scattering length: the effective range of the potential has to be increased essentially linearly with the scattering length. This does not seem to match the experimental conditions where the scattering length is adjusted by tuning the atomic reso- 
nances in an external magnetic field. The range of the inter-atomic interaction is then left essentially unchanged.

Instead, an attractive finite-range potential might be a more realistic interaction model for descriptions of trapped Bose gases with Feshbach resonances. Indeed, with an attractive potential an arbitrary large positive scattering length can be achieved by fine-tuning the energy of the bound two-body state, while maintaining the given realistic effective range.

However, attractive potentials with bound states bring in a major complication for numerical calculations: a large number of many-body self-bound negative-energy states appears in the system and the condensate state in the trap becomes a highly excited state.

For a homogeneous Bose gas an approximate Jastrowtype wave-function was employed where the paircorrelation function was essentially a solution of the twobody equation $[10,12]$. In contrast we propose a direct numerical diagonalisation of the many-body Hamiltonian where the condensate state of trapped bosons appears as a many-body excited state which is automatically orthogonal to all the self-bound negative-energy states.

The purpose of this paper is to investigate the energy and the condensate fraction of a system of trapped bosons with attractive potentials as function of the scattering length and the number of bosons, and compare the results with the zero-range and repulsive model.

\section{System and numerical techniques. -}

The system. We consider a system of $N$ identical bosons with mass $m$ and coordinates $\mathbf{r}_{i}, i=1, \ldots, N$, in a spherical harmonic trap with frequency $\omega$. The Hamiltonian of the system is given by

$$
H=-\frac{\hbar^{2}}{2 m} \sum_{i=1}^{N} \frac{\partial^{2}}{\partial \mathbf{r}_{i}^{2}}+\sum_{i<j} V\left(\left|\mathbf{r}_{i}-\mathbf{r}_{j}\right|\right)+\frac{m \omega^{2}}{2} \sum_{i=1}^{N} r_{i}^{2},
$$

where the system parameters are taken from [9]: $m=$ 86.909amu, $\omega=2 \pi \times 77.87 \mathrm{~Hz}$, the trap length $b_{t}=$ $\sqrt{\hbar /(m \omega)}=23095 \mathrm{au}$.

Two-body potentials. The zero-range potential model,

$$
V_{Z R}(r)=\frac{4 \pi \hbar^{2} a}{m} \delta(r),
$$

has only one length parameter, $a$. For dilute bosonic systems this parameter is customarily chosen to be equal the inter-atomic scattering length. The zero-range potential provides then the correct low-energy scattering amplitude in the first order Born approximation. The zerorange potential can only be used with an appropriate non-correlated functional space [13], like the Hartree-Fock product wave-functions. In the latter case it leads to the famous Gross-Pitaevskii equation [8].

For the finite-range potential model we use a Gaussian,

$$
V(r)=V_{0} \exp \left(-\frac{r^{2}}{b^{2}}\right),
$$

with the range $b=11.65 \mathrm{au}$ and a varied negative strength $V_{0}$. The variation of the strength is limited to the region where the potential provides exactly one two-body bound state and a positive scattering length.

Stochastic variational method. The wave-function of the system is represented as a linear combination of $K$ basis-functions taken in the form of symmetrised correlated Gaussian,

$$
\Psi=\hat{S} \sum_{k=1}^{K} C_{k} \exp \left(-\frac{1}{2} \sum_{i<j}^{N} \alpha_{i j}^{(k)}\left(\mathbf{r}_{i}-\mathbf{r}_{j}\right)^{2}\right),
$$

where $\hat{S}$ is the symmetrisation operator, and $C_{k}$ and $\alpha_{i j}^{(k)}$ are variational parameters. The linear parameters $C_{k}$ are determined by an ordinary diagonalisation of the Hamiltonian eq. (2) while the non-linear parameters $\alpha_{i j}^{(k)}$ are optimised stochastically by random sampling $[14,15]$ from a region that covers the distances from $b$ to $b_{t}$. The centerof-mass motion is assumed to be in the oscillator's ground state.

The zero-range potential eq. (3) requires an uncorrelated wave-function which we chose in the form of the linear combination of the hyper-radial basis-functions,

$$
\Psi_{\rho}=\sum_{k=1}^{K} C_{k} \exp \left(-\frac{1}{2} \alpha^{(k)} \rho^{2}\right),
$$

where $\rho^{2}=\sum_{i=1}^{N} r_{i}^{2}$ is the hyper-radius of the system. This function is totally symmetric and thus does not require the symmetrisation operator $\hat{S}$. The zero-range potential with the hyper-radial variational wave-function eq. (6) provides results similar to Gross-Pitaevskii equation [15].

The calculation of a highly excited state with the fully correlated basis eq. (5) is a difficult numerical task and is only possible for relatively small number of particles.

However for a typical system of trapped atoms even when the scattering length is large the density of the system remains small, $n b^{3} \ll 1$, and one can assume that only binary collisions play a significant role in the system's dynamics. In this approximation the variational wave-function can be simplified by only allowing two-body correlations in the basis-functions,

$$
\begin{aligned}
\Psi_{2 b} & =\hat{S} \sum_{k=1}^{K} C_{k} \\
& \times \exp \left(-\frac{1}{2} \alpha^{(k)} \rho^{2}-\frac{1}{2} \beta^{(k)}\left(\mathbf{r}_{1}-\mathbf{r}_{2}\right)^{2}\right),
\end{aligned}
$$

where $\alpha^{(k)}$ and $\beta^{(k)}$ are the nonlinear parameters. The symmetrisation of this function can be done analytically [15] which greatly simplifies the numerical calculations.

During the calculation of a given system the number of Gaussian in the basis is increased and the stochastic optimisation is carried out until the number of negative 
energy states and the energy of the lowest state with positive energy is converged. The convergence within four digits typically requires about $5 \times 10^{2}$ Gaussian and about $10^{5}$ random trials per nonlinear parameter. The stochastic optimisation algorithm is easily parallelisable with close to linear scalability.

Condensate fraction. We first calculate the one-body density matrix defined as

$$
\begin{aligned}
n\left(\mathbf{r}, \mathbf{r}^{\prime}\right) & =\int d \mathbf{r}_{2} \ldots d \mathbf{r}_{N} \\
& \times \Psi^{\star}\left(\mathbf{r}, \mathbf{r}_{2}, \ldots, \mathbf{r}_{N}\right) \Psi\left(\mathbf{r}^{\prime}, \mathbf{r}_{2}, \ldots, \mathbf{r}_{N}\right),
\end{aligned}
$$

where $\mathbf{r}_{i}$ are the coordinates of the atoms measured from the center of the trap. The density matrix is normalised to unity independent of the number of particles.

The density matrix is then diagonalised, meaning that its single-particle eigenfunctions $\chi_{i}(\mathbf{r})$ and the corresponding eigenvalues $\lambda_{i}$ are calculated,

$$
\int d \mathbf{r}^{\prime} n\left(\mathbf{r}, \mathbf{r}^{\prime}\right) \chi_{i}\left(\mathbf{r}^{\prime}\right)=\lambda_{i} \chi_{i}(\mathbf{r}) .
$$

The condensate fraction, $\lambda_{0}$, is then defined as the largest eigenvalue.

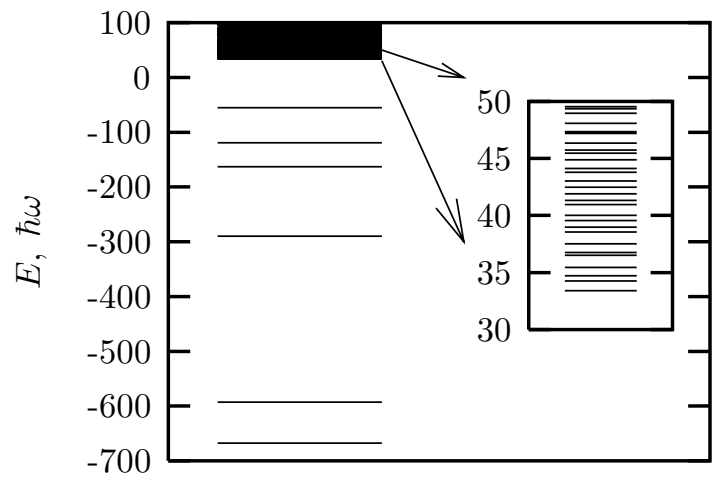

Fig. 1: The typical spectrum (in the vicinity of zero energy) of a system of $N=20$ bosons in an oscillator trap eq. (2) interacting via an attractive Gaussian two-body potential eq. (4) with one bound state and a positive scattering length. The inset shows the beginning of the quasi-continuum spectrum.

Bose-Einstein condensate state. For repulsive potential models it is simply the ground state of the trapped many-boson system that is identified as the Bose-Einstein condensate state (BEC-state).

With deep attractive two-body potentials, however, the many-body system in a trap has a large number of selfbound negative-energy states and identification of the BEC-state is not obvious.

The typical spectrum of a trapped many-body system with attractive potentials is shown on fig. 1. The system has a number of deeply bound states with negative energies and then a positive quasi-continuum spectrum which starts at about $\frac{3 N}{2} \hbar \omega$ and has the characteristic distance between levels of the order $\hbar \omega \ll \hbar^{2} /(2 m b)^{2}$.

Apparently the BEC-state should then be the lowest state of the quasi-continuum spectrum or, equivalently, the lowest state with positive energy.

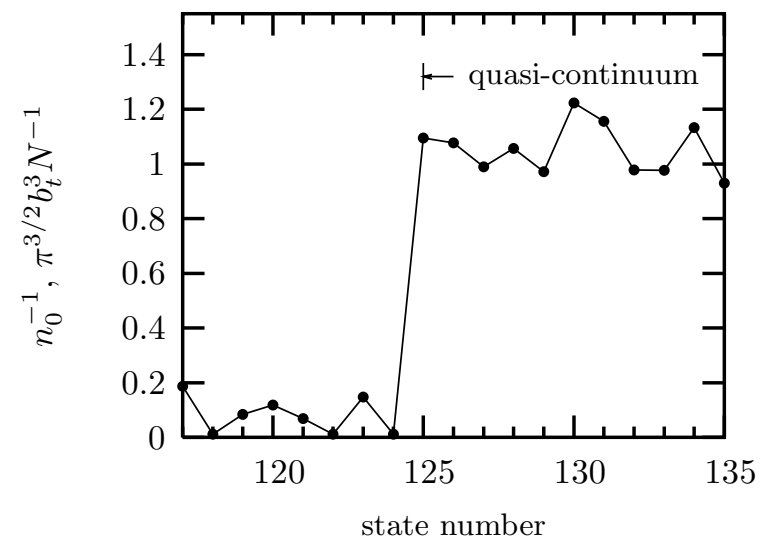

Fig. 2: The inverse central density $n_{0}^{-1}$ in oscillator units for a system of trapped bosons from fig. 1 as function of the state number. Shown are only the states in the vicinity of the beginning of the quasi-continuum spectrum.

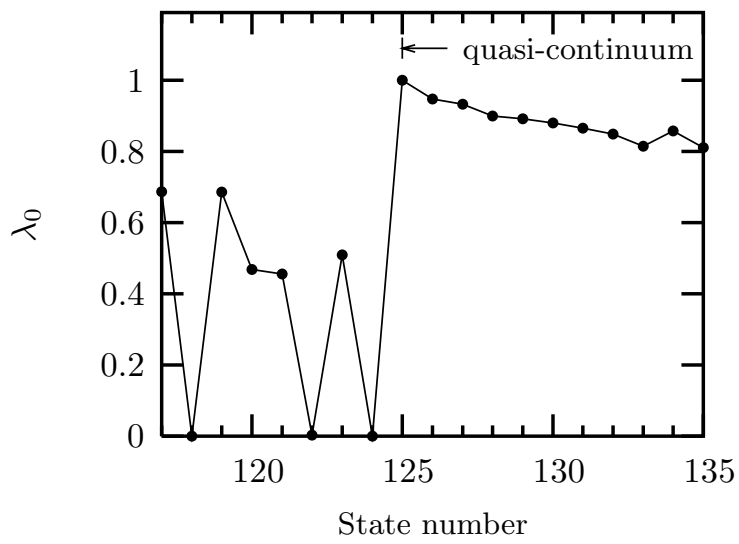

Fig. 3: The condensate fraction of a system of trapped bosons from fig. 1 as function of the state number. Shown are only the states in the vicinity of the beginning of the quasi-continuum spectrum.

To verify this assumption we calculate the central density, $n_{0}$, of the system for the negative- and positive-energy states around zero energy. The results are shown on fig. 2 in the form of the inverse central density (the volume per particle) $n_{0}^{-1}$. In the BEC-state the atoms should occupy the whole volume of the trap and thus the volume per particle should be close to one (in the correspondingly scaled oscillator units). The states with negative energy are selfbound states with much higher density and thus much smaller volume per particle. And indeed that is what the figure shows - a sharp increase in the volume per parti- 
cle from a small value to about unity exactly at the lowest state with positive energy where the quasi-continuum starts.

Another test is the very condensate fraction, shown for several states around zero energy on fig. 3. The self-bound states with negative energy must have smaller condensate fraction compared to the BEC-state, and the excitations from the BEC-state must gradually deplete the condensate fraction. Apparently this is what is seen on the figure - a sharp increase of the condensate fraction to about $100 \%$ at the lowest state with positive energy with the subsequent gradual depletion.

We have thus verified that in the case of attractive potentials the BEC-state of a system of trapped bosons is the lowest state with positive energy.

Table 1: The energies in units of $\hbar \omega$ for the BEC-state of a system of $N$ bosons in a harmonic trap eq. (2) for different interaction models with the same scattering length of $100 \mathrm{au}$. For the Gross-Pitaevskii (GP), hard-spheres (HS), and zerorange (ZR) models the BEC-state is the ground state; for the attractive model (A) the BEC-state is the lowest state with positive energy. The Gross-Pitaevskii and hard-spheres data are taken from [9]. The attractive model employed the twobody correlated basis eq. (7).

\begin{tabular}{|c|c|c|c|c|}
\hline$N$ & GP & HS & ZR & A \\
\hline \hline 3 & 4.51032 & $4.51036(2)$ & 4.5103 & 4.510 \\
\hline 5 & 7.53432 & $7.53443(4)$ & 7.5342 & 7.534 \\
\hline 10 & 15.1534 & $15.1537(2)$ & 15.1533 & 15.154 \\
\hline 20 & 30.638 & $30.640(1)$ & 30.6394 & 30.640 \\
\hline
\end{tabular}

Accuracy of the two-body correlated basis. In ref. [9] the energies of several low-density systems of trapped bosons were calculated using the Gross-Pitaevskii and repulsive hard-sphere models with the same "natural" scattering length of $100 \mathrm{au}$. In this regime the systems exhibit universality and the energies calculated in both models were very close.

To test the accuracy of our two-body correlated basis eq. (7) (which is expected to be a good approximation in the low-density regime) as well as the identification of the BEC-state for attractive potentials we consider the same systems with the same scattering length but with the attractive potential eq. (44) and calculate the energy of the BEC-state according to our prescription. The BEC-state is now an excited state and is identified in the calculations as the lowest state with positive energy. We also calculate the energies for the zero-range potential model eq. (3) with hyper-radial trial wave-function eq. (6).

The results are given in table 1. As expected, these low-density systems with relatively short scattering length exhibit universality as all potential models give essentially the same results. We conclude that we do a correct identification of the BEC-state and that that the two-body correlated basis has an adequate accuracy.
Table 2: The energies, in units of $\hbar \omega$, of the lowest state with positive energy for a system of 4 bosons in a harmonic trap eq. (2). The bosons interact via an attractive Gaussian potential eq. (4) with the strength $V_{0}$ and the scattering length $a$. The results from fully-correlated basis eq. (5) and from the two-body correlated basis eq. (7) are designated correspondingly $E$ (full) and $E(2 \mathrm{~b})$.

\begin{tabular}{|c|c|c|c|}
\hline$V_{0}, \mathrm{au}$ & $a, \mathrm{au}$ & $E(2 \mathrm{~b})$ & $E($ full $)$ \\
\hline \hline$-1.400 \mathrm{e}-7$ & 119.4 & 6.025 & 6.025 \\
\hline$-1.300 \mathrm{e}-7$ & 327.0 & 6.067 & 6.067 \\
\hline$-1.290 \mathrm{e}-7$ & 402.4 & 6.083 & 6.083 \\
\hline$-1.280 \mathrm{e}-7$ & 525.4 & 6.108 & 6.108 \\
\hline$-1.270 \mathrm{e}-7$ & 761.0 & 6.155 & 6.156 \\
\hline$-1.260 \mathrm{e}-7$ & 1400 & 6.282 & 6.283 \\
\hline$-1.255 \mathrm{e}-7$ & 2430 & 6.478 & 6.481 \\
\hline$-1.252 \mathrm{e}-7$ & 4370 & 6.818 & 6.848 \\
\hline$-1.251 \mathrm{e}-7$ & 5962 & 7.059 & 7.112 \\
\hline
\end{tabular}

To check the accuracy of the two-body correlated basis also for large scattering lengths we perform a test calculation for 4 particles with fully correlated and with two-body correlated basis for vastly different scattering lengths. The results are given in table 2 .

Although the accuracy of the two-body correlated basis decreases somewhat with the increase of the scattering length, yet the relative accuracy is better than $1 \%$ even for exceedingly large scattering lengths.

\section{Results. -}

Energy. Fig. 4 4shows the energy per particle for different $N$ and $a$ for two interaction models, zero-range potential and attractive potential. For small scattering lengths the different potential models give the same universal results - the system is model independent. For larger scattering lengths the energies from the attractive model are systematically below the zero-range model, quite unlike the repulsive model which goes above the zero-range model $[9,11]$. For very large scattering lengths the attractive model, unlike the zero-range and repulsive models, becomes insensitive to the scattering length and the energies converge to a constant. This is consistent with the Jastrow-type approximation of ref. [12].

In the regime close to the two-body threshold, where the scattering length is large, an arbitrary large change in the scattering length needs only an infinitesimally small change of the depth of the attractive potential (see table 2). Therefore when the scattering length is larger than the trap length it ceases to be a physical length scale for the system which is then only subjected to a very small change of the depth of the two-body potential which rapidly converges towards the two-body threshold value. Since the external oscillator potential turns all continuum states into discrete states all singularities due to various thresholds are removed. Then clearly an infinitesimally 


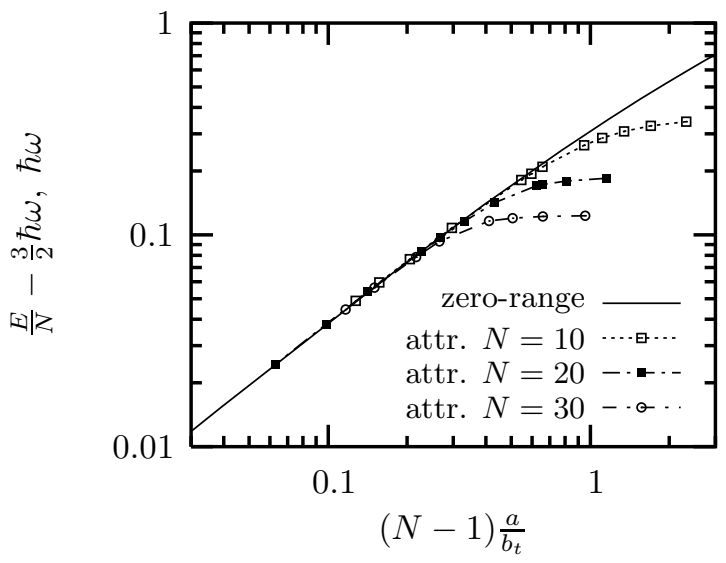

Fig. 4: The energy per particle $E / N$ as function of the scattering length $a$ for a BEC-state of a system of $N$ identical bosons in a harmonic trap eq. (2) for the zero-range model eq. (3) (solid line), and attractive potential model eq. (4) (broken lines). The zero-range results are $N$-independent in this parametrization.

small change in the potential, despite the large change in the scattering length, only leads to a linearly infinitesimal change in the system which then becomes independent on the scattering length.

On the contrary for the finite-range repulsive potential models an increase of the scattering length needs an almost proportional increase in the effective range of the potential. Thus the system never ceases to depend on the scattering length.

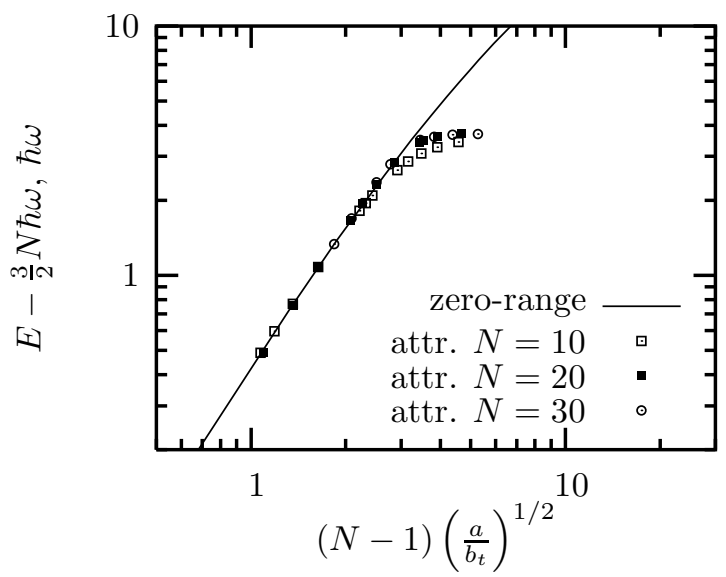

Fig. 5: The same as is in fig. 4 but plotted in a different parametrisation.

If we plot the data using a different parametrisation, namely $E$ as function of $(N-1)\left(\frac{a}{b_{t}}\right)^{1 / 2}$, the energy data points seem to follow a "universal" curve as shown on fig. [5]

Condensate fraction. Our results for the condensate fraction of a system of trapped identical bosons in a BECstate is shown on fig. 6. For small scattering lengths the

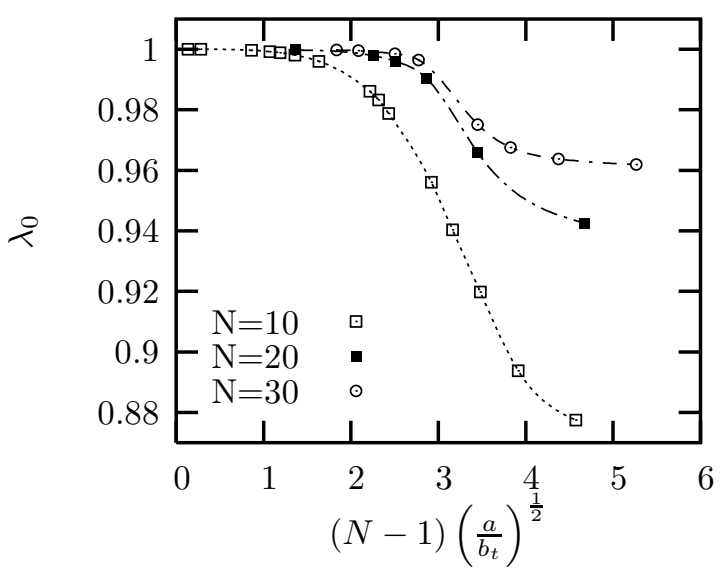

Fig. 6: The condensate fraction of a BEC-state of a system of $N$ identical bosons in a harmonic trap eq. (2) with attractive interparticle potential eq. (4) as function of the scattering length $a$.

system is $100 \%$ condensate. Then when $(N-1)\left(\frac{a}{b_{t}}\right)^{1 / 2}$ is about 3 the condensate fraction rapidly drops a few percent before stabilising again. Although we could not reach further due to numerical convergence problems, we expect that the condensate fraction will not appreciably change with the further increase of the scattering length. This seems consistent with the energies being stagnated when approaching the two-body threshold.

This behavior is qualitatively different from what happens within the repulsive models. In the hard-spheres Monte-Carlo simulations [11] the condensate fraction starts to deviate from $100 \%$ much later, when $\frac{a}{b_{t}}$ is of the order of 0.1 , and with further increase of the scattering length the condensate is completely quenched.

Again our results are different from the Jastrow-type approximation [12], where the condensate also becomes fully quenched. However the authors of ref. [12] believe that their estimates for the condensate fraction are "rather crude".

We have to note also that our wave-function includes only two-body correlations which may lead to an overestimate of the condensate fraction.

Conclusions. - We have calculated the energy and the condensate fraction of a system of $N$ bosons in a harmonic trap as function of the number of bosons and the scattering length $a$. Specifically we considered the regime where the scattering length is positive and not small compared to the trap length. The positive scattering length is modeled using an attractive two-body potential with a bound two-body state. The many-body system then has a large number of negative-energy self-bound states and the condensate in the trap is identified as the lowest excited state with positive energy.

When the scattering length is small compared to the trap length the system shows model independence (universality) - the results from the attractive potential model 
are very close to those from the zero-range and the repulsive potential models.

In the limit of large scattering length the system becomes independent of the scattering length, contrary to the zero-range and the repulsive models.

The condensate fraction decreases with the scattering length and reaches a finite constant at large scattering lengths contrary to the repulsive models where it reaches zero in this limit.

For the attractive potentials the energy per particle of the system of trapped bosons follows a universal curve.

$$
* * *
$$

Numerous fruitful discussions with H.H. Soerensen and T. Kjaergaard are acknowledged.

\section{REFERENCES}

[1] Ph. Courteille, R. S. Freeland, and D. J. Heinzen, Phys. Rev. Lett. 81 (1998) 69.

[2] E. Braaten, and H.-W. Hammer, Physics Reports 428 (2006) 259.

[3] L.H. Thomas, Phys. Rev. 47 (1935) 903.

[4] V.N. Efimov, Phys. Lett. 33B (1970) 563.

[5] C.R.C. Chen et al, Phys. Rev. C 44 (1991) 50; P.F. Bedaque, H.W. Hammer, U van Kolck, Nuclear Physics A 676 (2000) 357; D.V. Fedorov, and A.S. Jensen, Nucl.Phys. A 697 (2002) 783.

[6] E. Nielsen, and J.H. Macek, Phys. Rev. Lett. 83 (1999) 1566.

[7] E. Nielsen, D.V. Fedorov, A.S. Jensen, Phys. Rev. A 56 (1997) 3287.

[8] F Dalfovo, S Giorgini, LP Pitaevskii, and S Stringari, Rev.Mod.Phys. 71 (1999) 463.

[9] D. Blume, and C.H. Greene, Phys. Rev. A 63 (2001) 063601.

[10] S. Giorgini, J. Boronat, and J. Casulleras, Phys. Rev. A 60 (1999) 5129.

[11] J.L. DuBois and H.R. Glyde, Phys.Rev. A 63 (2001) 023602.

[12] S. Cowell et.al., Phys.Rev.Lett. 88 (2002) 210403.

[13] D.V. Fedorov and A.S. Jensen, Phys. Rev. A 63 (2001) 063608.

[14] Y. Suzuki, and K. Varga, Sthochastic Variational Approach to Quantum-Mechanical Few-Body Problems, (Springer-Verlag, Berlin, Heidelberg, 1998).

[15] H.H. Sørensen, D.V. Fedorov, and A.S. Jensen, AIP Conference Proceedings, 777 (2005) 12. 